\title{
Co-Channel Interference Cancellation at the User Terminal in Multibeam Satellite Systems
}

\author{
G. Cocco $^{\dagger}$, M. Angelone ${ }^{\Uparrow}$ and A.I. Perez-Neira ${ }^{1,2}$ \\ ${ }^{\dagger}$ German Aerospace Center - DLR \\ Oberpfaffenhofen, D-82234, Wessling, Germany \\ "European Space Agency - ESTEC, Noordwijk - The Netherlands \\ ${ }^{1}$ Centre Tecnològic de Telecomunicacions de Catalunya - CTTC \\ Parc Mediterrani de la Tecnologia, Av. Carl Friedrich Gauss 7 08860, Castelldefels - Spain \\ ${ }^{2}$ Department of Signal Theory and Communications \\ Universitat Politecnica de Catalunya, Barcelona, Spain \\ giuseppe.cocco@dlr.es, martina.angelone@esa.int, ana.perez@cttc.es
}

\begin{abstract}
We study the applicability of soft interference cancellation in the forward link of commercial multibeam satellite systems with focus on mobile terminals. We adopt a standard currently used in commercial satellite systems as a reference. The multibeam satellite antenna radiation diagram has been generated using a physical optics reflector model, while state-ofthe art channel models have been used for the land mobile satellite (LMS) channel. The interference pattern has been derived through a system simulator developed by the European Space Agency (ESA). Starting from the analysis of the interference pattern we study the application of a low complexity soft interference cancellation scheme. Our results show that, under realistic interference and propagation conditions and for existing standards, a two-colors frequency reuse scheme can be employed while guaranteeing service availability across the coverage and keeping the complexity at the user terminals relatively low.
\end{abstract}

\section{INTRODUCTION}

Bandwidth limitation is one of the main capacity-limiting factors in wireless communications systems. A common practice to overcome bandwidth shortage in both satellite and terrestrial networks using multiple beams/cells consists in dividing the available spectrum into sub-bands (colors) and reuse them over non-adjacent geographical regions. However, if on the one hand the reuse of frequency allows for a more efficient utilization of the spectral resources, on the other hand it determines an increase of the co-channel interference (CCI) due to the non-ideal antennas radiation patterns. Despite the improvements in antennas technology, undesired side lobes still constitute challenging problem in geostationary (GEO) satellite communications since the interference coming from co-channel beams can heavily affect the reception of the desired signal at the user terminal such that either the link throughput or the availability are penalized. This problem is exacerbated by the use of aggressive frequency reuse patterns. Interference cancellation techniques at the user terminal (UT) represent a possible solution to this problem. Many different interference cancellation techniques have been proposed up to date. A comprehensive overview is presented in [1]. A low complexity option for mitigating interference is to adopt a maximum a posteriori (MAP) symbol detector. Such detector has the drawback of having a complexity that grows expo- nentially with the number of signals to detect. In order to keep complexity low, trading part of the performance, several simplified schemes have been proposed in literature such as [2], [3] and [4]. Iterative decoding has been shown to achieve the multiple access channel (MAC) capacity in [5], by integrating error control coding with multiple access interference suppression. In [6] two iterative low complexity algorithms for adjacent channel interference (ACI) cancellation in satellite systems are presented. In [7] the authors proposed a parallel multi-user detector for adjacent channel interference cancellation in the return link of Inmarsat's Broadband Global Area Network (BGAN) system.

In the present paper we study the applicability of soft co-channel interference cancellation in the forward link of a satellite system with high frequency reuse based on a realistic scenario with focus on mobile terminals. The results presented here have been developed within the Advanced Research in Telecommunications Systems (ARTES) [8] project Next Generation Waveform for Increased Spectral Efficiency (NGWISE) founded by the European Space Agency (ESA) [9]. The standard adopted in the European Telecommunications Standard Institute (ETSI) Satellite Component of UMTS (SUMTS) [10] has been used as a baseline. Such standard is currently used in commercial satellite systems [11]. The multibeam satellite antenna radiation diagram has been generated through a commercial software used for satellite antenna design and analysis, while the interference pattern has been calculated using a system simulator developed by ESA. A stateof-the art channel model has been adopted for the land mobile satellite (LMS) channel. Unlike most of previous works, we start from the analysis of the interference distribution across the coverage area. Based on the interference distribution we propose a low complexity interference management solution. It is worth noting that our work differs from [6] and [7] in that co-channel rather than adjacent channel interference is considered. In fact, whenever standard channel spacing is considered $^{1}$ and an aggressive frequency reuse scheme is

\footnotetext{
${ }^{1}$ this may not be the case if techniques such as time-frequency packing are applied [12]. However, this falls out of the scope of the present work.
} 
applied, indeed CCI becomes the most relevant source of interference in the system as its level is much higher with respect to the ACI. Unlike in [7] we consider the forward link rather than the return link. Interference cancellation in the forward link is constrained by the complexity at the UT, especially within the LMS context. We show that, assuming a realistic interference spatial distribution, the optimal detector can be applied at the receiver with affordable complexity if an appropriate synchronization of the signals at the feeder stations and at the satellite is provided. Our results show that frame error rates as low as $10^{-3}$ can be achieved in the whole covered area while using a two-color frequency reuse scheme with a dramatic increase in spectral efficiency with respect to coloring schemes usually adopted in commercial satellite systems.

The rest of the paper is organized as follows. In Section II the system model is presented while in Section III we describe the proposed solution specifying the required modifications to ETSI standard [13]. The numerical results are presented in Section IV while Section V summarizes the main contributions of the paper.

\section{System Model}

Let us consider the forward link of an interactive geostationary (GEO) multibeam satellite system with 210 user-link beams operating in L/S band. Each beam occupies half of the available user-link bandwidth and a two-color frequency reuse pattern is adopted, with a single polarization per beam.

Due to the satellite antenna radiation pattern each beam suffers from the interference generated by the closest cochannel beams. For a frequency-reuse of two, ten co-channel beams can be identified as the most significant contributors to the total interference. The geographical position of reference and interfering beams is shown in Figure 1, where the red color identifies the reference beam while orange is used for the co-channel interfering beams. In order to be representative of the best case and the worst case scenarios we considered two beams for our analysis, namely beam 105 and beam 110, one at the center and one at the edge of the global coverage, respectively.

Given a UT in a given beam we refer to the desired signal as reference signal. Single antenna terminals are considered.

The received signal at time $t$ when $N_{\text {int }}$ interferers are present is:

$$
\begin{aligned}
y(t) & =h(t)\left[g^{C}(t) x^{C}(t)\right. \\
& \left.+\sum_{n_{\text {int }}=1}^{N_{\text {int }}} g_{n_{\text {int }}}^{I}\left(t_{n_{\text {int }}}\right) x_{n_{\text {int }}}^{I}\left(t_{n_{\text {int }}}\right)\right]+n(t),
\end{aligned}
$$

where $t_{n_{\text {int }}}=t-\tau_{n_{\text {int }}}, \tau_{n_{\text {int }}}$ being the time offset of interferer number $n_{\text {int }}$, while

$$
g_{n_{\text {int }}}^{I}(t)=G_{n_{\text {int }}}^{I} e^{j\left(2 \pi \Delta \nu_{n_{i n t}} t+\varphi_{n_{i n t}}\right)},
$$

$G_{n_{\text {int }}}^{I}$ being the antenna gain of the co-channel interfering beam $n_{\text {int }}$ in the direction of the UT, normalized to the gain of the reference signal, while $\Delta \nu_{n_{i n t}}$ and $\varphi_{n_{i n t}}$ are the frequency

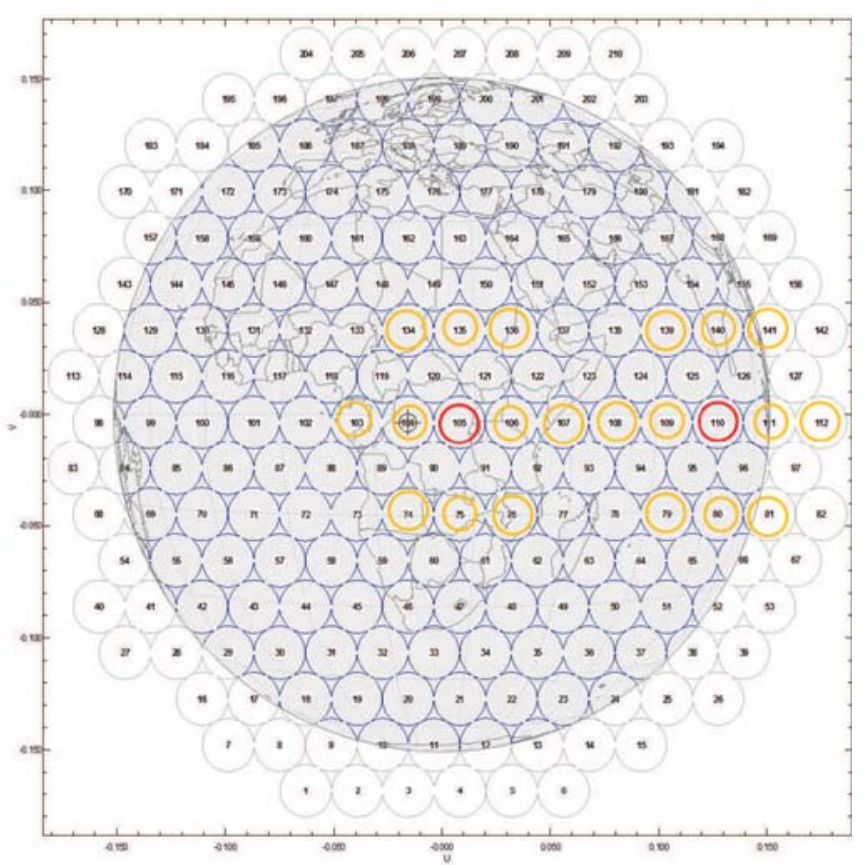

Fig. 1. Considered reference and interfering beams and conventional numbering. Reference and interfering beams are shown in red and yellow, respectively.

and phase offsets with respect to the local oscillator at the UT, respectively. Similarly we defined

$$
g^{C}(t)=G^{C} e^{j\left(2 \pi \Delta \nu_{C} t+\varphi_{C}\right)}
$$

with $G^{C}=1$.

Signals $x^{C}(t)$ and $x_{n_{\text {int }}}^{I}(t), n_{\text {int }} \in\left\{1, \ldots, N_{\text {int }}\right\}$, are the reference and the interfering signals, respectively. The interfering signals (and similarly the desired one) can be expressed as

$$
x_{n_{i n t}}^{I}(t)=\sum_{l=1}^{N_{N_{i n t}}^{C W}} s_{n_{i n t}}(l) g\left(t-l T_{s}^{n_{i n t}}\right),
$$

where $g(t)$ is a root-raised cosine pulse with roll-off $\alpha, s_{n_{\text {int }}}(l)$ represents the $l-t h$ received symbol from interferer $n_{\text {int }}$, $T_{s}^{n_{i n t}}$ is the symbol duration while $N_{n_{i n t}}^{C W}$ is the number of modulated symbols in a codeword for interferer $n_{\text {int }}$. $h(t)$ takes into account the channel effect (phase rotation and propagation loss). Note that $h(t)$ is a common multiplying factor for all signals, since all waveforms originate from the same spacecraft and in a forward link system all signals cover the same path to the UT. We assume that the maximum frequency offset is such that $\Delta \nu_{n_{\text {int }}} T_{S} \ll 1 / 100, \forall n_{\text {int }} \in\left\{1, \ldots, N_{\text {int }}\right\}$. The sample taken at time $t_{k}$ after matched filtering and 
sampling of signal $y(t)$ is:

$$
\begin{aligned}
y_{k} & =h\left(t_{k}\right)\left[g^{C}\left(t_{k}\right) s(k)\right. \\
& \left.+\sum_{n_{\text {int }}=1}^{N_{\text {int }}} g_{n_{\text {int }}}^{I}\left(t_{k}^{n_{\text {int }}}\right) \sum_{l=1}^{N_{n_{\text {int }}}^{C W}} s_{n_{\text {int }}}(l) g\left(t_{k}^{n_{\text {int }}}-l T_{s}^{n_{\text {int }}}\right)\right]+w_{k},
\end{aligned}
$$

where $t_{k}^{n_{\text {int }}}=t_{k}-\tau_{n_{\text {int }}}$ while $w_{k}$ 's are independently and identically distributed (i.i.d.) zero mean complex Gaussian random variables with variance $\sigma^{2}$ in each component. The interfering signals gains $G_{n_{i n t}}^{I}$ are determined by the satellite antenna radiation pattern. The use of a realistic antenna pattern is of fundamental importance for the selection and the performance assessment of an adequate interference cancellation technique at the UT. In the following we give details about the antenna pattern and system model adopted in the present paper.

\section{A. System Simulations and Antenna Pattern Models}

This section describes the system simulator developed by ESA and used to compute the interference pattern as well as the models used to create the considered antenna pattern.

The ESA satellite communication systems analysis tool, developed in MATLAB, performs a multi-dimensional spacetime link budget over a uniform latitude-longitude grid of users, averaging over a user-defined set of time availabilities with the related channel attenuations and availability probabilities. The reference propagation models are based on ITU recommendation [14] and it is assumed that the traffic request across different beams is uniform. For the sake of this study we focus on clear sky conditions, since atmospheric attenuation does not represent a serious impairment in L/S band. Each user of the grid is assigned to a specific beam if the gain of such beam in its location is the highest across the coverage. Then, based on the frequency plan and on the consequent beam coloring, the resulting interference pattern and distribution are calculated. The simulated system foresees the use of Adaptive Coding and Modulation (ACM) that enables each user to select the most efficient modulation and coding (ModCod) scheme allowed by the link condition. In general the ACM in LMS systems is more challenging with respect to the case of fixed terminals due to the rapid changes in the communication channel induced by the terminal motion. In [13] a return channel is used to feed-back the measured SNR (or SINR) to the Bearer Control Layer. The information is used at the control unit to select the bearer according to a target QoS. Such system is used to adapt the communication rate to the long-term channel variations only, since short-term fading is covered by the link margin [13, Section 7]. Further analysis in the implementation of the ACM mechanism is out of the scope of this paper.

The down-link signal to interference ratio in the point $x$ belonging to beam $i$ is given by:

$$
\left(\frac{C}{I}\right)_{c o}^{D L}(x)=\frac{P_{T X \_S A T}(i)+G_{T X_{c o-p o}}^{s a t}(i, x)}{\sum_{j=1}^{N_{c o-c h}} P_{T X_{-} S A T}(j)+G_{T X_{c o-p o}}^{s a t}(j, x)},
$$

where:

- $P_{T X_{-} S A T}(i)$ is the saturated power per carrier of beam $i$

- $G_{T X_{c o-p o}}^{s a t}(i, x)$ is the co-polar satellite TX antenna gain of beam $\mathrm{i}$ in the location $x$

- $P_{T X_{-} S A T}(j)$ is the saturated power per carrier of beam $\mathrm{j}$; note that in the analysis it has been assumed that all the carriers have equal power and therefore this term can be assumed to be a constant

- $G_{T X_{\text {co-po }}}^{s a t}(j, x)$ is the co-polar satellite TX antenna gain of co-channel beam beam $j$ in the location $x$.

We assume that solid state power amplifiers (SSPAs) are used on-board the satellite payload. As in this analysis we focus on the first $N_{c o}=10$ strongest interferers received at the user terminal we further define for each of them:

$$
\left(\frac{C}{I_{j}}\right)_{c o}(x)=\frac{G_{T X_{c o-p o}}^{s a t}(i, x)}{G_{T X_{c o-p o}}^{s a t}(j, x)}
$$

as the signal to co-channel interference related to the $j$-th cochannel interferer, assuming that $I_{j} \geq I_{j+1} \forall j \in\left\{1, \ldots, N_{c o}\right\}$ and $I_{N_{c o}+1}=0$. As for the considered antenna pattern, a commercial software for antenna design analysis and coverage planning has been used to reproduce a beam pattern similar to the one of the commercial system BGAN [11], which adopts the ETSI standard [13]. The software is based on physical optics reflector modeling and allows for accurate characterization of the directivity of both the co-polar and the cross-polar fields, as well as scan-aberrations and losses [15]. A geostationary satellite in the 25 deg East orbital position has been considered.

Finally, the reflector has been modeled with the parameters listed in Table I.

\section{Proposed Solution}

We consider the forward bearers family of the standard [13]. We aim to find an interference cancellation solution at the UT that is at the same time efficient and that has low complexity. We propose to split the complexity between system and UT levels. In the following the modifications required at system level with respect to the standard are detailed.

TABLE I

SATELLITE REFLECTOR PARAMETERS.

\begin{tabular}{|c|c|}
\hline Parameter & Value \\
\hline Aperture size $[\mathrm{m}]$ & 9 \\
\hline F/D & 1.34 \\
\hline Beam spacing/ $\theta$ 3dB [deg] & 1.363 \\
\hline Crossover Level [dB] & -3 \\
\hline Aperture Efficiency & $59.1 \%$ \\
\hline Directivity [dBi] & 40.85 \\
\hline
\end{tabular}




\section{A. System Level}

The modifications that would be required to [13] are hereafter specified and the related implications and feasibility discussed.

1) It is assumed that the symbol rate $R_{s}^{I}=1 / T_{s}^{I}$ of the strongest interferer is the same as that of the reference signal $R_{s}^{C}=1 / T_{s}, T_{s}$ being the symbol period of the reference signal. Although in principle different channel code rates, modulations and FEC block sizes may be used in the two signals, the simulation results we present in Section IV show that there are some restrictions to the modulations and code rates that can be adopted. Note that assuming the same symbol rate for the reference and the interfering signals implies $T_{s}^{n_{\text {int }}}=T_{s}, \forall n_{\text {int }} \in$ $\left\{1, \ldots, N_{\text {int }}\right\}$, in expression (5).

2) The symbols of reference and interfering signals are aligned such that the intersymbol interference (ISI)-free sample instants of the reference signal correspond to the ISI-free sample instants of the interferer, which implies $\tau_{n_{\text {int }}}=0 \forall n_{\text {int }} \in\left\{1, \ldots, N_{\text {int }}\right\}$ in expression (5). However, in Section IV we show that this constraint can be relaxed up to a certain extent.

3) The receiver knows the modulation used by the interferer. This information can be made available to the UT through the global beam and using knowledge of the user position, which is currently foreseen in [13] through GPS signal. Knowing the position with respect to the reference beam, a user could derive which is the strongest interfearing beam. The information about the modulation used in each beam (and thus also in the interfearing beam) during a given time slot is transmitted over the global beam. We use this assumption as it simplifies the description of the proposed scheme, although in Section IV we will show that it can be actually removed.

\section{B. MUD at the User Terminal}

We assume that the channel of both the reference signal and the strongest interferers as well as the ISI-free sample instants of the reference signal can be estimated. This assumption is usually taken in most multi-user detection (MUD) systems. Channel estimation can be performed using the pilot symbols inserted at regular intervals in the frame as foreseen in [13]. In case the pilot symbols of reference and interfering signal overlap, joint estimation methods may be adopted (e.g., E-M algorithm [16] $)^{2}$. An extensive literature is available on the subject and further analysis is out of the scope of this paper. We further assume that conditions $1 \rightarrow 3$ of Section III-A hold.

In case no interference is present, in a typical receiver the turbo decoder is fed with the log-likelihood ratio (LLR) vector of the sampled received signal. The $j$-th component, $j \in\left\{1, \ldots, R N_{C}^{c w}\right\}$ of the LLR vector for QPSK signalling

\footnotetext{
${ }^{2}$ A similar problem has been addressed in [17] where the feasibility of the joint estimation of phase, amplitude and frequency offsets of five colliding signals is studied.
}

and using the Grey mapping scheme of [10] can be expressed as:

$$
L L R_{j}=\log \left(\frac{\operatorname{Pr}\left\{b_{j}=1 \mid y_{k}\right\}}{\operatorname{Pr}\left\{b_{j}=0 \mid y_{k}\right\}}\right)=\log \left(\frac{P_{k, s_{2}}+P_{k, s_{3}}}{P_{k, s_{0}}+P_{k, s_{1}}}\right),
$$

for $j=2 k-1$, while

$$
L L R_{j}=\log \left(\frac{P_{k, s_{1}}+P_{k, s_{3}}}{P_{k, s_{0}}+P_{k, s_{2}}}\right),
$$

for $j=2 k$, where $P_{k, s_{n}}$ is the probability to observe the sample $y_{k}$ conditioned to the transmission of the symbol $s_{n}$, $n \in\{0,1,2,3\}$, while $b_{j}$ indicates the $j$-th coded bit in the transmitted codeword. Equation (8), and similarly Equation (9), is derived taking into account that, according to the considered mapping, symbols $s_{2}$ and $s_{3}$ correspond to a bit pair with the first bit equal to 1, while the first bit of the pair mapping to $s_{0}$ and $s_{1}$ is equal to 0 . Equation 8 can be easily extended to the case of 16 QAM modulation. The probability $P_{k s_{n}}$ is proportional to:

$$
P_{k, s_{n}} \propto \exp \left\{\frac{\left|y_{k}-h\left(t_{k}\right) G^{C} s_{n}\right|^{2}}{2 \sigma^{2}}\right\} .
$$

In the case of a single interferer with constellation size $M$, the probability that the $k$-th symbol of the reference signal $s(k)$ is equal to $s_{n}$ can be expressed as:

$$
P_{k, s_{n}}=\sum_{m=0}^{M-1} P_{s_{m}^{I}} P_{k, s_{n}, s_{m}^{I}}
$$

where $P_{k, s_{n}, s_{m}^{I}}$ represents the probability to receive $y_{k}$ conditioned to symbols $s_{n}$ and $s_{m}^{I}$ in the reference and in the interfering signals, respectively, while $P_{s_{m}^{I}}$ represents the probability of transmitting symbol $s_{m}^{I}$, which is assumed to be equal to $1 / M$. The probability $P_{k, s_{n}, s_{m}^{I}}$ is proportional to:

$P_{k, s_{n}, s_{m}^{I}} \propto \exp \left\{\frac{\left|y_{k}-h\left(t_{k}\right) g^{C}\left(t_{k}\right) s_{n}-h\left(t_{k}\right) g_{n_{i n t}}^{I}\left(t_{k}\right) s_{m}^{I}\right|^{2}}{2 \sigma^{2}}\right\}$

This can be easily extended to the case of a generic number of interferers $N_{\text {int }}$ each with its phase and frequency offsets and amplitude, leading to the following expression for the optimal symbol detector,

$$
P_{k, s_{n}}=\sum_{m_{1}=0}^{M_{1}-1} \cdots \sum_{m_{N_{i n t}}=0}^{M_{N_{i n t}}-1} \prod_{j=1}^{N_{i n t}} P_{s_{j}^{I}} P_{k, s_{n}, s_{m_{1}}^{I}, \ldots, s_{m_{N_{i n t}}}^{I}},
$$

where $M_{n_{\text {int }}}$ is the constellation size of interferer number $n_{\text {int }}$. The complexity of expression (13) grows exponentially with the number of interferers.

Once the a-priori probabilities for the desired signal have been derived they can be used to calculate the L-values to be fed to the turbo decoder. In this case the only modification at the receiver side with respect to the standard terminal is limited to the signal detector, while no modification would be needed at the decoder. The performance of the receiver in terms of FER (and potentially in terms of throughput, as higher ModCods could be adopted) can be further improved through an iterative detection-decoding scheme, which would, 
on the other hand, increase the receiver complexity and require further modifications to the existing structure of the receiver described in [10]. Such possibility is not further discussed here for a matter of space.

\section{NumericAl REsults}

In the following we evaluate the performance of the proposed algorithm for the scenario described in Section II.

We start by describing in detail the reference scenario and the interference distribution generated through the system level simulator presented in SectionII. The beam numbering and geographical location are those shown in Fig. 1. In Table II, at the bottom of the page, we show the $C / I$ related to each of the 10 strongest interferers for both beam 105 and 110 in two points, namely at the center of the beam $(\mathrm{CoB})$ and at the edge of the beam (EoB).

\begin{tabular}{|r|r|r|r|r|}
\hline & & & & \\
\hline & 8 & 9 & 10 & \\
\hline & & & & \\
\hline 4 & 5 & & 6 & 7 \\
\hline & & & & \\
\hline & 1 & 2 & 3 & \\
\hline & & & & \\
\hline
\end{tabular}

Fig. 2. Interference pattern and conventional numbering of the co-channel beams. The central red rectangle represents the reference beam, while the yellow rectangles represent the ten strongest interfering beams.

The relative numbering of the interferers is given according to Figure 2, where the central rectangle represents the reference beam while the yellow rectangles represent the strongest co-channel interferers.

With reference to Table II at the bottom of next page, it can be seen that in the EoB cases the power of the interferer number 5 is comparable to that of the reference signal while the second strongest interferer is attenuated more than 11 $\mathrm{dB}$. On the other hand, in the CoB the strongest interferer is at least $12 \mathrm{~dB}$ lower than the reference signal. Let us consider the worst case scenario, i.e., the EoB. In this case there is only one strong interferer plus nine interferers with a relatively weak power, that, by the Central Limit Theorem, can be modeled as Gaussian noise. Trying to apply MUD to these low-power interferers is not likely to have a relevant impact on the system performance while it would increase significantly the complexity of the receiver. A better choice is to apply the MUD to the desired signal and the strongest interferer while treating the rest of the interferers as noise. In order to understand whether the assumption of having at most one significant interferer is realistic in each point of the beam footprint, we analyzed the distribution of the total $C / I$ across the whole beam. The distribution is shown in Fig. 3, where three cases have been considered for each point in the two beams: i) all the interferers are present (top-left), ii) only the first strongest interferer has been removed (bottom-left) iii) the first two strongest interferers have been removed (bottomright). From the figure it can be seen that the total $C / I$ reaches negative values, in logarithmic scale, in some areas of the beam (e.g., in the EoB points considered in the table shown
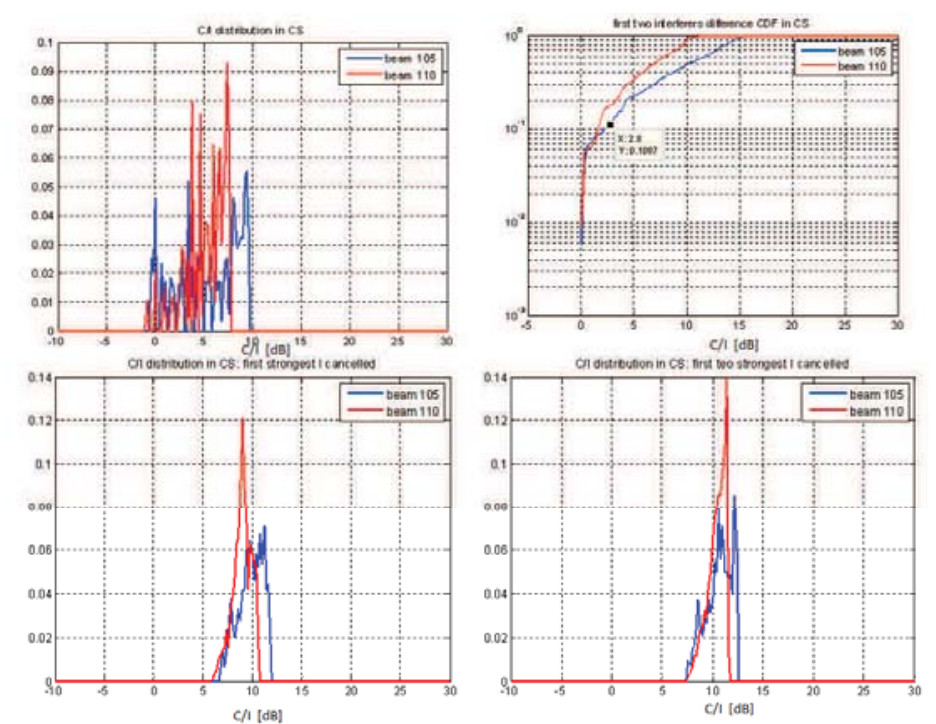

Fig. 3. Probability density function (PDF) of interference across the covered area (horizontal axis represents $C / I$, expressed in $\mathrm{dB}$ ) in case: 1 ) all interferers are present (top-left), 2) the strongest interferer has been removed (bottom-left), 3) the two strongest interferers have been removed (bottomright). The cumulative distribution function (CDF) of the difference between the two strongest interferers across the beam is also shown (top-right).

in table II) when all the interferers are present. Removing the strongest interferer determines a minimum $C / I$ larger than or equal to $6 \mathrm{~dB}$ in any point of the two considered beams. We further notice that the cancellation of the second strongest interferer further increases the minimum $C / I$ of only about 1-1.5 dB. From the analysis of Fig. 3 we conclude that the total $\mathrm{C} / \mathrm{I}$ is mainly limited by the first strongest interferer while the second one has only limited impact on performance. We propose therefore to deal with only one interfering signal while treating the others as noise in order to keep the complexity low. The detector described in Section III. We recall that this implies that the only modification needed at the decoder side is in the detector, for which the a-priori probability in case of one interferer reduces to:

$$
P_{k, s_{n}}=\sum_{m=0}^{M-1} \exp \left\{\frac{\left|y_{k}-h g^{C}\left(t_{k}\right) s_{n}-h g^{I}\left(t_{k}\right) s_{m}^{I}\right|^{2}}{2 \sigma_{e q}^{2}}\right\},
$$

$M$ being the cardinality of the interferer's constellation. The correspondent block scheme is shown in Fig. 4.

In order to take into account the influence of the other interferers (which reduces the reliability of the detection) in the received signal's statistics we increase $\sigma_{e q}^{2}$ by several $\mathrm{dBs}$ (6 in the following simulations) with respect to the actual variance of the thermal noise $\sigma^{2}$. The optimal choice would be to choose the value of $\sigma_{e q}$ by estimating the noiseplus-interference power. However, in practice keeping a fixed value of the variance can be a good compromise since i) the thermal noise component can be either given by the terminal manufacturer or easily estimated, while the power due to residual interference may not be easy to measure, as the 


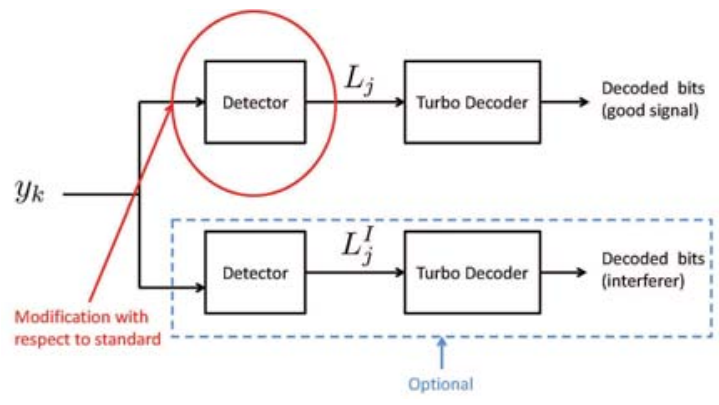

Fig. 4. Proposed SIC scheme. Only the strongest interferer is taken into account and no iterative detection is applied (i.e., reference signal is detected as described in Section III and decoded using the BGAN turbo decoder) Optionally also the strongest interfering signal can be decoded.

received signal is made up by the sum of the (strong) reference signal, a (possibly strong) dominant interferer and the residual interferer (estimation of the residual interference power in such conditions would increase the complexity of the receiver) and ii) we observed that the FER shows little sensibility to the exact value of $\sigma_{e q}$. In the simulations presented in the following the signal model described in equations 1-5 has been adopted: all 10 interferers have been simulated including channel code, modulation and channel effect, and scaling the powers according to Table II. We first present the results obtained in AWGN channel and then those for the LMS scenario. The simulation setup for the two cases is depicted in Fig. 5. The simplified scheme shown in Fig. 4 (i.e., the received signal passes through the detector and through the turbo decoder just once) has been used.

\section{A. AWGN Channel}

In figures 6,7 and 8 we show the FER curves for the considered MUD using the interference pattern detailed in Table II. Different combinations of MODECODs available in the standard [13] have been used, namely QPSK rate $1 / 3$ for all signals in Fig. 6, QPSK with rate $2 / 5$ for all signals in Fig. 7 and QPSK with rate $1 / 3$ for the reference signal and 16 QAM rate $1 / 3$ for interferers in Fig. 8.

From the plots it emerges that the target FER of $10^{-3}$ can be achieved using QPSK modulation in all signals up to rate $2 / 5$ while if 16 QAM is used in one of (or both) the signals the target FER cannot be achieved for values of $C / N$ of practical interest.

In the following subsection we present the results for an LMS scenario.

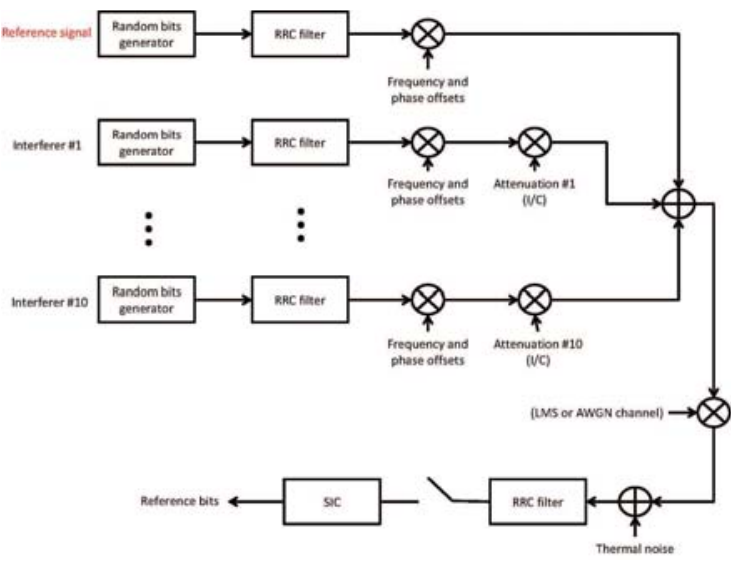

Fig. 5. Simulation setup in AWGN and LMS channels.

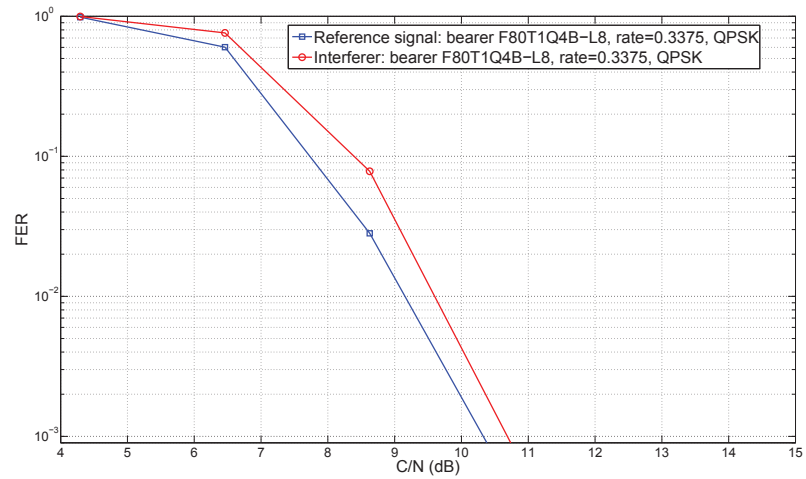

Fig. 6. FER in AWGN with MUD. Bearer F80T1Q4B-L8 (QPSK rate $1 / 3$, symbol rate $33600 \mathrm{sym} / \mathrm{sec}$, roll-off 0.25 ) is used for all signals. The interference pattern for beam $110 \mathrm{EoB}$ detailed in Table II (worst case scenario) has been used.

\section{B. LMS Channel}

The channel model used in the simulations presented in the following is a land-mobile satellite (LMS) channel for vehicles moving at a speed of $50 \mathrm{kmph}$ in a suburban environment. A channel realization of 30 minutes $(25 \mathrm{~km}$ path at $50 \mathrm{kmph})$ has been used, corresponding to about $2.7 \cdot 10^{4}$ FEC blocks for bearer F80T025Q1B-L8 (QPSK, rate 1/3, symbol rate 8400 symbols per second). The time series has been generated using an LMS channel generator implementing the Perez-Fontan model [19].

TABLE II

TABLE WITH FOUR SAMPLES OF THE INTERFERENCE PATTERN. EACH ROW CONTAINS THE $C / I$ RELATED TO THE TEN STRONGEST INTERFERING SIGNALS FOR EITHER A CENTER-OF-BEAM (BEST LOCATION) POINT OR AN EDGE-OF-BEAM (WORST LOCATION) POINT IN BEAMS 105 (BEST BEAM) AND 110 (WORSt BEAM). The tOTAL $C / I$ IS Also REPORTED FOR EACH CASE [18].

\begin{tabular}{|c|c|c|c|c|c|c|c|c|c|c|c|c|}
\hline & \multirow{2}{*}{\multicolumn{10}{|c|}{$C / I[\mathrm{~dB}]$}} & \multirow{3}{*}{$C / I$ Total $[\mathrm{dB}]$} \\
\hline & & & & & & & & & & & & \\
\hline Beam & & 1 & 2 & 3 & 4 & 5 & 6 & 7 & 8 & 9 & 10 & \\
\hline \multirow{2}{*}{105} & $\mathrm{CoB}$ & 37.1107 & 21.5885 & 32.1618 & 37.2214 & 17.9294 & 14.5272 & 27.3961 & 31.9697 & 20.7406 & 29.2564 & 11.46467 \\
\hline & EoB & 15.6046 & 15.5337 & 29.8048 & 15.8007 & 0.3881 & 15.1211 & 21.4581 & 44.3936 & 38.1297 & 21.875 & -0.17835 \\
\hline \multirow{2}{*}{110} & $\mathrm{CoB}$ & 30.3903 & 19.541 & 32.9636 & 35.0503 & 13.7636 & 12.1374 & 21.4154 & 29.4771 & 18.9879 & 30.8531 & 8.607253 \\
\hline & EoB & 27.2207 & 29.9124 & 22.4402 & 17.9726 & 0.1185 & 11.5821 & 18.8873 & 14.2254 & 15.2343 & 27.9627 & -0.6047 \\
\hline
\end{tabular}




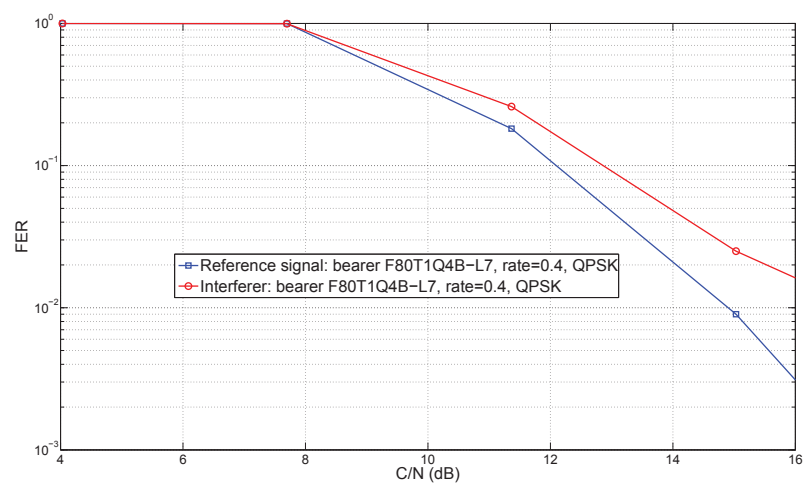

Fig. 7. FER in AWGN with MUD. Bearer F80T1Q4B-L7 (QPSK rate $2 / 5$, symbol rate $33600 \mathrm{sym} / \mathrm{sec}$, roll-off 0.25 ) is used for all signals. The interference pattern for beam $110 \mathrm{EoB}$ detailed in Table II (worst case scenario) has been used.

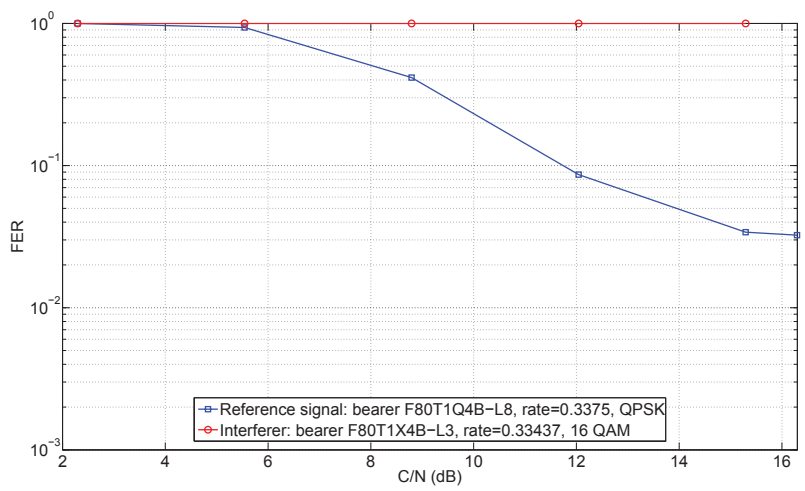

Fig. 8. FER in AWGN with MUD. Bearer F80T1Q4B-L8 (QPSK rate $1 / 3$, symbol rate $33600 \mathrm{sym} / \mathrm{sec}$, roll-off 0.25 ) is used for the reference signal while bearer F80T1X4B-L3 (16 QAM rate 1/3, symbol rate 33600 symbols per second, roll-off 0.25 ) is used for the interferers. Note that rate $1 / 3$ is the lowest code rate available in BGAN. The interference pattern for beam 110 EoB detailed in Table II (worst case scenario) has been used.

In Fig. 9 we show the frame error rate for the reference signal using the proposed simplified SIC scheme. 10 interferers have been considered using the $C / I$ values in Table II. Bearer F80T025Q1B-L8 (QPSK, rate 1/3) of standard [13] has been adopted for all signals.

Fig. 9 shows that the SIC scheme reaches the target FER of $10^{-3}$ in all the considered cases, showing a neat enhancement with respect to the case in which no interference cancellation is applied. Thus it can be seen that decoding is possible in all considered points, while it is not feasible without the MUD algorithm. A relatively high $C / N$ is required in order to fulfill FER requirements in EoB which is due partly to the challenging propagation scenario. As a matter of facts it can be seen in Fig. 9 that, even in case no interference is present in the system, a $C / N$ of about $14 \mathrm{~dB}$ is needed to reach a target FER of $10^{-3}$. We also note that the FER obtained in the LMS channel in case of no interference is almost the same as that in CoB. This is because the total

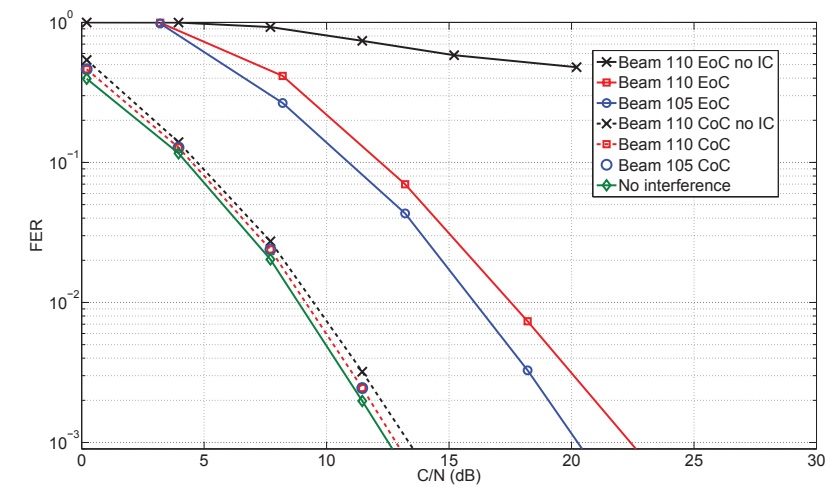

Fig. 9. Frame error rate for the reference signal using the simplified SIC scheme with one iteration (one detection and one decoding iteration). A 30 minutes LMS channel series in suburban environment generated according to [20] has been used. 10 interferers have been considered using the $C / I$ values in Table II. Bearer F80T025Q1B-L8 (QPSK, channel code rate $1 / 3$, symbol rate 8400 symbols per second) of standard [10] has been adopted for all signals.

interference level in $\mathrm{CoB}$ is low enough to allow for correct decoding even without SIC, which justifies the fact that the same performance is achieved by the SIC and the no IC (no interference cancellation) schemes.

An important outcome of the simulations is that the system results to be interference-limited mainly in the EoB area, while interference has little effect in the CoB area. We also showed that dealing with a single interferer is enough to make decoding possible. We emphasize that these results have been achieved with a limited increase in the receiver complexity, as only the demapper has been modified with respect to the receiver described in [10]. The fact that a $C / N$ larger than $20 \mathrm{~dB}$ is needed in EoB could be addressed by using a code with longer codewords (the turbo code of DVB-SH, for instance, has codewords which are an order of magnitude larger than those used in the simulations just presented), an interleaver with an adequate depth or a combination if the two, compatibly with memory and latency constraints in the user terminals.

\section{COnCLusions}

We studied the application of co-channel soft interference cancellation in multibeam mobile satellite systems with a twocolour frequency reuse scheme. We took the ETSI standard [13], currently used in commercial satellite systems, as a reference and simulated the beam radiation and the interference patterns using a realistic antenna model. The calculation of the interference pattern has been carried using simulator developed by ESA. Due to strong complexity limitations in mobile terminals, we proposed to move part of the complexity to the system level, by aligning signals transmitted over different beams and adding specific signalling information in the global beam. We started from the analysis of the interference levels 
across the beams selecting two of them as best and worst case scenarios. In order to keep the complexity at the receiver low, we proposed a simplified scheme in which only the detector is modified with respect to the standard [10]. Our results showed that even under challenging propagation conditions and with strong interference, the considered scheme leads to interesting results, achieving a target FER of practical interest. We showed that the proposed approach may constitute a concrete possibility to live together high levels of interference with a relatively limited increase in complexity at both system and user level. This comes at the expense of a limitation in the ModCods that can be used.

\section{ACKNOWLEDGEMENTS}

The present work has been carried out under the ARTES 1 programme founded by the European Space Agency. The view expressed herein can in no way be taken to reflect the official opinion of the European Space Agency.

The research leading to these results has received funding from the Spanish Ministry of Science and Innovation under projects TEC2011-29006-C03-02 (GRE3N-LINK-MAC) and the Catalan Government (2009SGR0891).

\section{REFERENCES}

[1] J. G. Andrews, "Interference cancellation for cellular systems: a contemporary overview," IEEE Wireless Comm., vol. 12, no. 2, pp. 19-29, 2005.

[2] X. Wang and H. V. Poor, "Iterative (turbo) soft interference cancellation and decoding for coded CDMA," IEEE Transactions on Comm., vol. 47 no. 7, pp. 1046-1061, 1999.

[3] M. Kopbayashi, J. Boutros, and G. Caire, "Successive interference cancellation with SISO decoding and EM channel estimation," IEEE Journal on Selected Areas in Comm., vol. 19, no. 8, pp. 1450-1460, 2001.

[4] G. Colavolpe, D. Fertonani, and A. Piemontese, "SISO detection over linear channels with linear complexity in the number of interferers," IEEE Journal of Selected Topics in Signal Processing, vol. 5, no. 8, pp. 1475-1485, 2011.

[5] C. Schlegel, "Achieving the multiple-access capacity of the AWGN channel with iterative processing," in AESS European Conference on Satellite Telecommunications (ESTEL), Rome, Italy, Oct. 2012.
[6] B. F. Beidas, H. El-Gamal, and S. Kay, "Iterative interference cancellation for high spectral efficiency satellite communications," IEEE Transactions on Comm., vol. 50, no. 1, pp. 31-36, 2002.

[7] M. Moher, W. Zhang, P. Febvre, and J. Rivera-Castro, in Advanced Satellite Multimedia Systems conf. (ASMS) and Signal Processing for Space Communications workshop (SPSC).

[8] European Space Agency (ESA), "Advanced Research in Telecommunications Systems (ARTES),” http://telecom.esa.int/telecom/.

[9] European Space Agency, "Next generation waveform for improved spectral efficiency," http://telecom.esa.int/telecom/, 2013.

[10] European Telecommunications Standards Institute, "Satellite componen of UMTS (S-UMTS) family SL satellite radio interface; part 2: Physical layer specifications; sub-part 1: Physical layer interface," May 2012.

[11] Inmarsat, "Broadband Global Area Network (BGAN)," http://www.inmarsat.com/services/bgan.

[12] A. Barbieri, D. Fertonani, and G. Colavolpe, "Time-frequency packing for linear modulations: spectral efficiency and practical detection schemes," IEEE Transactions on Communications, vol. 57, no. 10, pp. 2951-2959, 2009.

[13] European Telecommunications Standards Institute, "Satellite component of UMTS (S-UMTS) family SL satellite radio interface; part 1: General specifications; sub-part 3: Satellite radio interface overview," May 2011.

[14] International Telecommunications Union - Radiocommunications Sector, "Propagation data and prediction methods requred for the design of Earth-space telecommunication systems: methods requred for the design of Earth-space telecommunication systems," Reccomendation ITU-R P.618-10 (10/2009), Oct. 2009.

[15] Satsoft, http://www.satsoft.com/

[16] M. Feder and E. Weinstein, "Parameter estimation of superimposed signals using the EM algorithm," IEEE Trans. on Acoustics, Speech and Signal Processing, vol. 36, no. 4, pp. 477-489, Apr. 1988.

[17] G. Cocco, N. Alagha, C. Ibars, and S. Cioni, "Practical issues in multiuser physical layer network coding," in IEEE Advanced Satellite Mobile Systems conf. (ASMS), Baiona, Spain, Sep. 2012.

[18] European Space Agency, "NGWISE project technical Annex B: Technologies, models and requirements for the work of RG2 on MSS," 2013.

[19] F. Perez-Fontan, M. A. Vazquez-Castro, S. Buonomo, J. P. PoiaresBaptista, and B. Arbesser-Rastburg, "S-band LMS propagation channel behaviour for different environments, degrees of shadowing and elevation angles," IEEE Trans. on Broadcasting, vol. 44, no. 1, pp. 40-76, Mar. 1998.

[20] F. Perez-Fontan, A. Mayo, D. Marote, R. Prieto-Cerdeira, P. Marino, F. Machado, and N. Riera, "Review of generative models for the narrowband land mobile satellite propagation channel," Int'l Journal of Satellite Comm. and Networking, vol. 26, pp. 291-316, 2008. 